Check for updates

Cite this: RSC Adv., 2018, 8, 33368

\title{
Oxide removal and stabilization of bismuth thin films through chemically bound thiol layers $\uparrow$
}

\author{
Giuseppe Alessio Verni, (D) abc Brenda Long, (D) *abc Farzan Gity, (D) b Martin Lanius, ${ }^{\mathrm{d}}$ \\ Peter Schüffelgen, ${ }^{d}$ Gregor Mussler, ${ }^{d}$ Detlev Grützmacher, ${ }^{d}$ Jim Greer (D) be \\ and Justin D. Holmes ${ }^{\text {abc }}$
}

\begin{abstract}
Bismuth has been identified as a material of interest for electronic applications due to its extremely high electron mobility and quantum confinement effects observed at nanoscale dimensions. However, it is also the case that Bi nanostructures are readily oxidised in ambient air, necessitating additional capping steps to prevent surface re-oxidation, thus limiting the processing potential of this material. This article describes an oxide removal and surface stabilization method performed on molecular beam epitaxy (MBE) grown bismuth thin-films using ambient air wet-chemistry. Alkanethiol molecules were used to dissolve the readily formed bismuth oxides through a catalytic reaction; the bare surface was then reacted with the free thiols to form an organic layer which showed resistance to complete reoxidation for up to 10 days.
\end{abstract}

Received 15th August 2018

Accepted 10th September 2018

DOI: $10.1039 / \mathrm{c} 8 \mathrm{ra06840b}$

rsc.li/rsc-advances

a semiconducting channel between semi-metallic source and drain regions, in what is defined as a confinement modulated gap transistor (CMGT). ${ }^{9}$ This is a dopant-free transistor that does not suffer from dopant fluctuation and activation problems. Recently a hetero-dimensional rectifier was formed on bismuth thin-films and consisted of a 3D semi-metallic region next to a thin $2 \mathrm{D}$ semiconducting region. ${ }^{10}$ Rectifying behaviour was observed in the Bi diode at room temperature, highlighting that a 'junction' can be created without dopant atoms.

However, the fabrication of the Bi CMGTs relies on the formation of the metal-semimetal junction in a UHV system in order to prevent surface re-oxidation, as the surface has been shown to readily re-oxidise when in contact with air, leading to a decrease in resistance. ${ }^{8}$ While UHV oxide removal through RIE is a simple process, it does not allow for surface passivation and subsequent ambient air handling, requiring an additional capping step to prevent re-oxidation of the device.

The oxidation of bismuth thin films was first reported by Hapase et al. ${ }^{11}$ in 1967, who found that oxidation proceeds by diffusion of the Bi from the thin film through the oxide film, as previously hypothesised, ${ }^{12}$ via a Wagner mechanism. Further studies then correlated the oxide thickness to a diffusion equation and showed that a metastable crystal phase of the oxide is present which evolves into a different stable phase upon heating applied. ${ }^{13}$ In 1990 Puckrin et al. ${ }^{14}$ compared the oxidation of bulk bismuth and thin films using different spectroscopy techniques and showed that, in both cases, $\mathrm{BiO}$ is the first oxide formed on the surface of the samples with a total thickness of approximately 8 monolayers $(c a .2 \mathrm{~nm})$. Further results showed that prolonged air oxidation times lead to further oxidation of the film and to the formation of $\mathrm{Bi}_{2} \mathrm{O}_{3}$, which ties 
back with the results described previously by Kowalczyk et al. ${ }^{15}$ Despite these reports, the oxidation behavior of Bi thin films has not been studied extensively.

Self-assembled monolayers (SAMs) formed by chemisorption of alkyl thiols on the surface of various inorganic substrates have been extensively reported in the literature; in particular, their use as corrosion inhibitors has been explored on various semiconductors ${ }^{16-19}$ and metals. ${ }^{2021}$ There are two examples of alkyl thiol adsorption on bulk Bi electrode surfaces; Adamovski et $a .^{22}$ reported the characterization of alkyl thiol adsorption thickness via the diffusion blocking properties of the layer, whilst Romann et al. ${ }^{23}$ characterized the formation of bismuth thiolate at the surface of the electrode in the absence of a selfassembled monolayer.

This article reports the first example of SAM formation on $\mathrm{Bi}$ thin films via a wet chemical process (Scheme 1). The SAM surfaces have been characterised via XPS, IR and AFM and show that, in a similar fashion as for $\mathrm{Cu}$ surfaces, ${ }^{21}$ the alkyl thiol plays an active role in removing the native bismuth oxide from the thin film surface and leads to subsequent stabilization of Bi surfaces preventing complete re-oxidation. Whilst complete oxide removal was not achieved for the films, likely due to a non-continuous passivating layer, the research highlights the ability to remove oxide and passivate $\mathrm{Bi}$ surfaces outside of a vacuum environment.

Bi films used in this study (supplied by the research centre, Jülich, in Germany) were grown by molecular beam epitaxy (MBE) on undoped Si [111] substrates. Prior to bismuth deposition, the Si substrates were chemically cleaned by the HF-last $\mathrm{RCA}^{24}$ procedure to remove the native oxide and to passivate the surface with hydrogen. The substrates were subsequently heated in situ to $700{ }^{\circ} \mathrm{C}$ for $20 \mathrm{~min}$ to desorb the hydrogen atoms from the Si surface. The Bi material flux was generated by an effusion cell operated at a temperature of $550{ }^{\circ} \mathrm{C}$, which yields a growth rate of $17 \mathrm{~nm} \mathrm{~h}^{-1} .8$

A round bottom flask was preheated using a heating mantle up to $180^{\circ} \mathrm{C}$ and left at this temperature for $30 \mathrm{~min}$. The Bi thin films were then placed in the flask under $\mathrm{N}_{2}$, annealed for $1 \mathrm{~h}$ and left to cool to room temperature. Solutions of 1-dodecanethiol (Sigma-Aldrich, $\geq 98 \%$ ) in isopropanol (IPA) were prepared, with concentrations ranging from 1 to $100 \mathrm{mM}$. The solutions were degassed using three freeze-pump-thaw cycles and transferred in the sample flask using a cannula, thus preventing any contact with the outer atmosphere.

(a) Anneal Bi at $180^{\circ} \mathrm{C}$ for 30 $\min$.

(b) Immerse in solution of DT overnight.
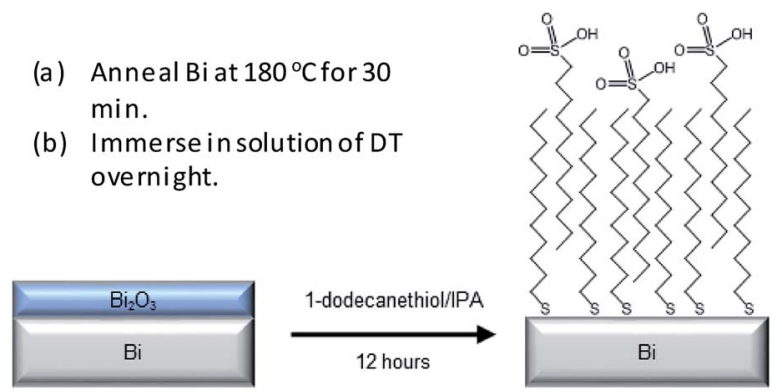

Scheme 1 Depiction of the procedure for the removal of bismuth oxide and its subsequent passivation with thiols.
The Bi samples were left immersed in solution at room temperature overnight after which time the solution was vacuum pumped out of the flask using a cannula. The samples were then dried by gently heating the flask using a heat gun in order to evaporate the remaining solvent. Clean IPA was pumped into the sample flask to rinse any adsorbed molecules. IPA was then removed, and the thin film dried once again. The same reaction procedure was also used on Bi powder (Fisher Scientific, 100 mesh, 99.5\% metal basis) which was used as received. The samples were then transferred to a $\mathrm{N}_{2}$ glovebox from the flask to a gel box which was subsequently transferred to a $\mathrm{N}_{2}$ filled sample preserver. The gel box was kept in the sample preserver until X-ray photoelectron spectroscopy (XPS) measurements were carried out. The exposure time during the loading procedure into the XPS entry chamber was less than $30 \mathrm{~s}$. The XPS powder samples were prepared by depositing the reacted powder on some carbon tape. The powder was degassed overnight in the XPS entry chamber before being analyzed. The exposure time to air of these samples was less than $1 \mathrm{~h}$. XPS data were collected using an Oxford Applied Research Escabase ${ }^{\circledR}$ XPS system. Survey scans were recorded between $0-1400 \mathrm{eV}$ with a step size of $0.7 \mathrm{eV}$, dwell time of $0.5 \mathrm{~s}$ and pass energy of $100 \mathrm{eV}$. Core level scans were acquired with a step size of $0.1 \mathrm{eV}$, dwell time of $0.5 \mathrm{~s}$ and pass energy of $20 \mathrm{eV}$ averaged over 20 scans. A non-monochromated Al-k $\alpha$ X-ray source operating at $100 \mathrm{~W}$ power was used for all scans. All spectra were acquired at a take-off angle of $90^{\circ}$ with respect to the analyser. Energy calibration for all spectra was obtained using the $\mathrm{C}$ 1s line at $284.8 \mathrm{eV}$ as reference data was processed using CasaXPS software where a Shirley background correction was employed. Relative sensitivity factors used for quantification purposes are from the CasaXPS software library. XPS thickness measurements were performed following the methodology originally defined by Cumpson et al., ${ }^{25}$ (see eqn (1)).

$$
\ln \left(\frac{I_{\mathrm{o}} S_{\mathrm{o}}}{I_{\mathrm{s}} S_{\mathrm{s}}}\right)-\left(\frac{\lambda_{\mathrm{o}}}{\lambda_{\mathrm{s}}}\right) \frac{1}{\lambda_{\mathrm{o}} \cos \theta}-\ln 2=\ln \sinh \left(\frac{t}{2 \lambda_{\mathrm{o}} \cos \theta}\right)
$$

where $I_{\mathrm{O}}$ and $I_{\mathrm{S}}$ represent the respective measured peak intensities of the overlayer (alkyl thiol molecules) and thin film peaks, $S_{\mathrm{o}}$ and $S_{\mathrm{s}}$ refer to the relative sensitivity factors for the overlayer and the thin film respectively. $\lambda_{\mathrm{o}}$ and $\lambda_{\mathrm{s}}$ are the attenuation lengths of electrons in the overlayer and the thin film. $\theta$ is the emission angle with respect to the surface normal. To minimise the effect of potential errors arising from surface roughness and inelastic scattering, a photon emission angle of $35^{\circ}$ was used in conjunction with a $90^{\circ}$ take-off angle with respect to the sample normal. The peak intensity of the overlayer peak, $I_{\mathrm{o}}$, and the peak intensity of the thin film peak, $I_{\mathrm{s}}$, were determined using CasaXPS software after a transmission correction. The relative sensitivity factors for the thin film peak $S_{\mathrm{s}}$ and the overlayer peak $S_{\mathrm{o}}$ were also obtained from the CasaXPS library and manually inputted into the data processing software to remove instrumental factors which may affect quantification. The local electron attenuation length (EAL) in the overlayer, $\lambda_{\mathrm{o}}$, was estimated, using the NIST Electron Effective Attenuation Length database, ${ }^{26}$ to be $2.1 \pm 0.2 \mathrm{~nm}$ for the $\mathrm{Bi}$ 4f component, and $1.7 \pm 0.2 \mathrm{~nm}$ for the $\mathrm{Bi} 4 \mathrm{~d}_{5 / 2}$ 
component. The surface was thoroughly cleaned by prolonged sonication in anhydrous solvents prior to characterization to remove all physisorbed species prior to analysis and to minimise contributions from contaminants to the overlayer thickness.

${ }^{1} \mathrm{H}(300 \mathrm{MHz})$ and ${ }^{13} \mathrm{C}(75.5 \mathrm{MHz})$ Nuclear Magnetic Resonance (NMR) spectra were recorded on a Bruker Advance 300 $\mathrm{MHz}$ NMR spectrometer. All spectra were recorded at $300 \mathrm{~K}$ in deuterated chloroform $\left(\mathrm{CDCl}_{3}\right)$ using tetramethylsilane (TMS) as an internal standard. Attenuated total reflectance infrared (ATR-FTIR) spectra were recorded using a Nicolet 6700 Infrared Spectrometer with a VariGATR and a liquid cooled MgCdTe detector using 3000 scans at a resolution of $2 \mathrm{~cm}^{-1}$. Spectra were collected under p-polarisation in an ambient atmosphere. Fig. 1 shows XPS scans of the Bi 4 f core level peak for the thin films before and after reaction with different concentrations of thiol solutions. At $156.5 \mathrm{eV}$ and $161.8 \mathrm{eV}$ the $\mathrm{Bi}$ metal $4 \mathrm{f}$ components are present ${ }^{27}$ and at $158.6 \mathrm{eV}$ and $163.4 \mathrm{eV} \mathrm{Bi}-\mathrm{X} 4 \mathrm{f}$ components $^{28}$ are present, where $\mathrm{X}$ is a heteroatom, in this case O. Treatment of the Bi films with a $1 \mathrm{mM}$ 1-dodecanethiol solution did not reduce the intensity of the $\mathrm{Bi}-\mathrm{X}$ peak considerably, as also shown in Table S1. $\dagger$ However, XPS analysis of Bi films treated with 10 and $100 \mathrm{mM}$ thiol solutions displayed a high Bi metal content, when the Bi peaks at $156.5 \mathrm{eV}$ and $161.8 \mathrm{eV}$ are compared to those of the as received sample. The shoulder peaks at $158.6 \mathrm{eV}$ and $163.4 \mathrm{eV}$, whilst usually associated with $\mathrm{Bi}_{2} \mathrm{O}_{3}$, in this case are likely to be composed of both $\mathrm{Bi}-\mathrm{O}$ and by $\mathrm{Bi}-\mathrm{S}$ components. This hypothesis is based on the analysis of the thickness of the thiol-coated Bi thin films by XPS following the method outlined in the experimental section. The thickness of the overlayer, calculated using the $\mathrm{Bi}-\mathrm{X}$ shoulder as the overlayer peak and the $\mathrm{Bi} 4 \mathrm{f}_{7 / 2}$ peak as the thin film peak, was approximately $0.95 \pm 0.10 \mathrm{~nm}$. The thickness of the oxide calculated using the $\mathrm{O} 1 \mathrm{~s} \mathrm{Bi}_{2} \mathrm{O}_{3}$ component as the overlayer peak and the $\mathrm{Bi} 4 \mathrm{~d}_{5 / 2}$ peak as the thin film peak was found to be $0.43 \pm 0.05 \mathrm{~nm}$. This discrepancy in thickness is likely due to $\mathrm{S}$

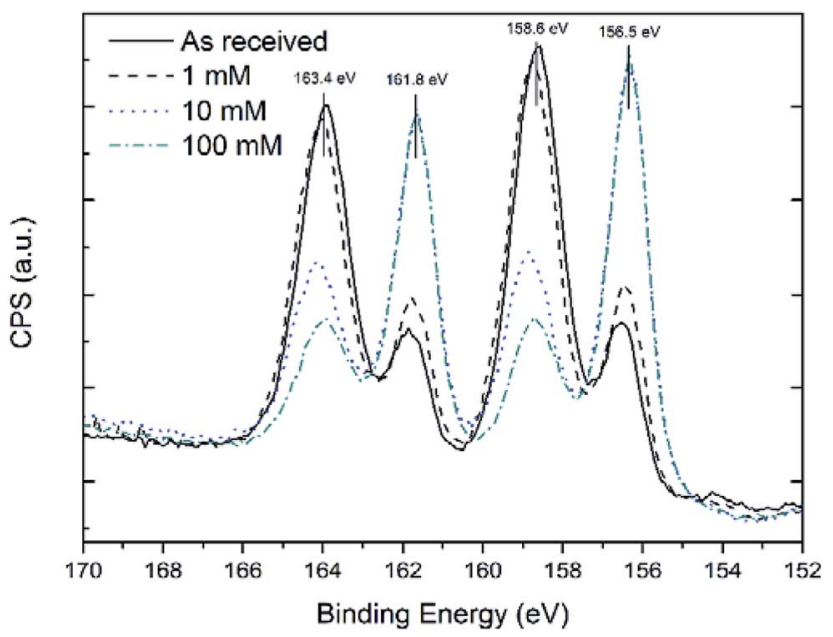

Fig. 1 Overlaid XPS spectra of $\mathrm{Bi} 4 \mathrm{f}$ core level after reaction with 1dodecanethiol solutions at different concentrations. Graphs have been normalised to the maximum of the as-received sample to underline the oxide reduction effect. also bound to surface $\mathrm{Bi}$ atoms, thus leading to a $\mathrm{Bi}$ 4f shoulder peak that was more intense due to contributions from both components, i.e. $\mathrm{Bi}-\mathrm{S}$ and $\mathrm{Bi}-\mathrm{O}$.

Fig. 2 shows an ATR-FTIR scan of the Bi thin film surface before and after the functionalisation process. The solventcleaned as-received $\mathrm{Bi}$ thin films presents vibrations only due to adventitious carbon adsorbed on the surface. After functionalisation and air exposure, multiple vibrations were observed at 2993 and $2909 \mathrm{~cm}^{-1}$, arising from C-H stretches and vibrations from the aliphatic carbon chains of the thiol molecules. In the fingerprint region between $1500-750 \mathrm{~cm}^{-1}$ there are the two peaks associated with the asymmetric stretching of $\mathrm{SO}_{2}$ at $1367 \mathrm{~cm}^{-1}$ and $1174 \mathrm{~cm}^{-1}$ and one peak due to symmetric stretching at $1083 \mathrm{~cm}^{-1} .^{29}$ This data suggests that the outer facing thiols from the second adsorbed layer, due to the oxidative environment created by the $\mathrm{Bi}_{2} \mathrm{O}_{3} / \mathrm{Bi}$ pair, ${ }^{30,31}$ are readily oxidised to their sulfonic acid equivalents. Surface bound thiols are known to progressively oxidise to sulfonic acids in the presence of strong oxidative environments. ${ }^{32,33}$

Bi(III) likely catalyses the formation of a disulphide bond between two 1-dodecanethiol molecules, similarly to what has already been observed for copper ${ }^{34}$ with water as a by-product.

As the sample/solution system is kept under $\mathrm{N}_{2}$, the oxide layer cannot form again and is consumed. ${ }^{30}$ Once the bare $\mathrm{Bi}$ surface is exposed, the thiol passivates the surface as the Bi-S bond is thermodynamically more favourable than the $\mathrm{Bi}-\mathrm{O}$ bond. ${ }^{35,36}$ To confirm this mechanism, further experiments were undertaken on Bi powder. The powder samples, which presented a bismuth oxide : bismuth ratio similar to that of the films (see Table $\mathrm{S} 1 \dagger$ ), were annealed at $180{ }^{\circ} \mathrm{C}$ for $1 \mathrm{~h}$, under similar conditions to the Bi-coated Si substrates. The annealed powder samples were then immersed in 1-dodecanethiol solutions made in IPA and left in air in order to promote reoxidation. After approximately $3 \mathrm{~h}$, the solutions changed colour from colourless to yellow, and a precipitate was formed which was collected and dissolved in $\mathrm{CDCl}_{3}$ for $\mathrm{NMR}$ analysis (See Fig. S1 $\dagger$ ). NMR data for the as-received 1-dodecanethiol was also collected and used for comparison to check for the presence of starting material at the end of the reaction.

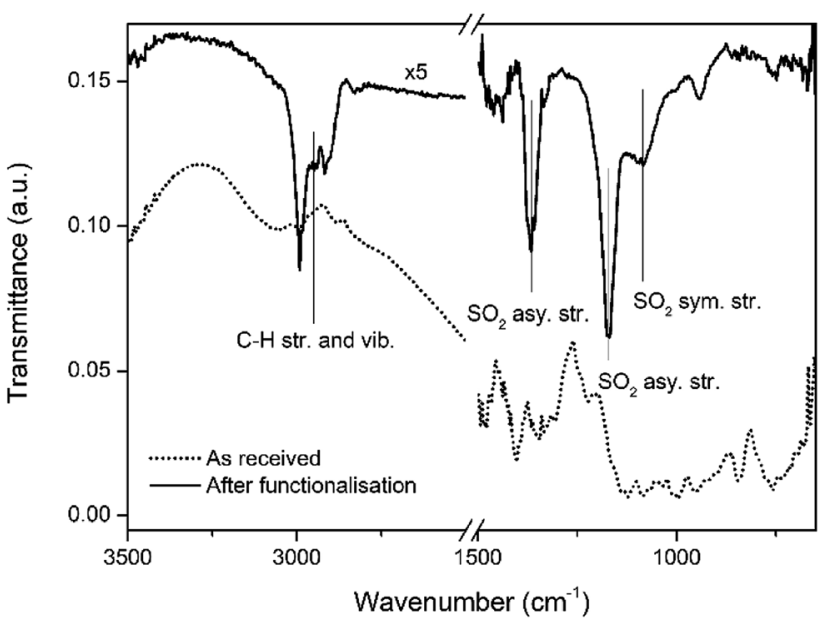

Fig. 2 IR spectra of Bi surface before and after functionalisation. 


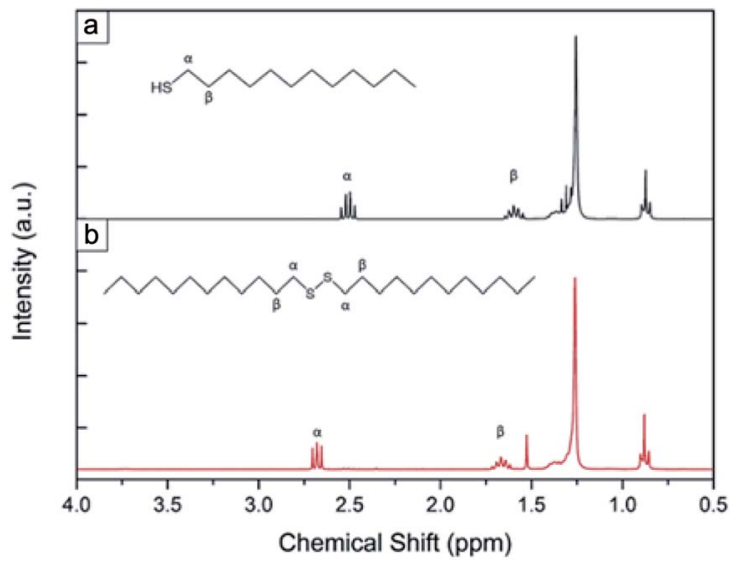

Fig. 3 NMR spectra of (a) the as-received 1-dodecanethiol used for the reaction and $(b)$ the dodecyldisulfide product found after reacting 1-dodecanethiol solution with bismuth powder.

Fig. 3(a) shows an NMR spectrum for the as-received 1dodecanethiol; a quartet resonating at $2.51 \mathrm{ppm}(J=7.33 \mathrm{~Hz})$, arising from the $\alpha$-methylene group of the thiol group was identified. The $\beta$-methylene and the thiol proton resonate at $1.60 \mathrm{ppm}$ (quintet, $J=7.58 \mathrm{~Hz}$ ) and $1.39 \mathrm{ppm}$ (unresolved multiplet) respectively, the methyl of the $\mathrm{C} 12$ carbon is found at $0.87 \mathrm{ppm}$ (triplet, $J=6.90 \mathrm{~Hz}$ ) and the remaining protons on C3C11 form an unresolved multiplet at 1.26 ppm. ${ }^{37}$ Fig. 3(b) shows an NMR spectrum of the reaction product formed upon reacting 1-dodecanethiol with Bi powder; two $\alpha$-methylene groups from a disulphide moiety resonate as a triplet at $2.68 \mathrm{ppm}(J=7.35$ $\mathrm{Hz}$ ); two $\beta$-methylene groups resonate at $1.67 \mathrm{ppm}$ (quintet, $J=$ $7.31 \mathrm{~Hz}$ ) and between 1.26 and $1.39 \mathrm{ppm}$ there is an unresolved multiplet formed by the protons on the two alkyl chains. The remaining two methyl groups at the end of the chains resonate at $0.88 \mathrm{ppm}$ (triplet, $J=6.70 \mathrm{~Hz}$ ). ${ }^{38}$ No starting material was observed in the final NMR spectra, as the characteristic $\alpha$-methylene quartet was not apparent. The reaction carried out with the
Bi powder in air can proceed indefinitely until all the starting material is oxidized; the powder is in fact allowed to re-oxidize, unlike under $\mathrm{N}_{2}$, thus catalysing the reaction. No precipitate or change in colour of the solution was observed when thiol passivation was undertaken on Bi thin films left in air, probably due to the much smaller surface area of $\mathrm{Bi}$ on a compared to the powder form, limiting the oxidation of a noticeable quantity of thiols. This might also explain why at low thiol concentrations only a small reduction of oxide was observed, as most of the thiols in solution are probably oxidized while removing the outer oxide layer, leaving only few molecules available to passivate the Bi films. Using concentrations higher than $100 \mathrm{mM}$ led to visible corrosion of the surface of the sample; therefore, the optimum level was found to be $10 \mathrm{mM}$ as it gave the highest oxide reduction without affecting surface quality.

Functionalised Bi thin film samples were then left in air and re-measured using XPS to check the extent of re-oxidation over time. Fig. 4(a) shows a comparison of the Bi $4 \mathrm{f}$ core level peaks of a $100 \mathrm{mM}$ functionalised sample after $1 \mathrm{~h}, 2$ days and 10 days of air exposure. The increase in intensity of the shoulder peaks at $159.3 \mathrm{eV}$ and $164.6 \mathrm{eV}$ arises from oxidation of exposed areas of the thin film, due to the non-continuity of the thiol film formed, as shown by AFM (see Fig. S2 $\dagger$ ). Fig. 4(b) shows the O 1s peak of thiol functionalised samples before and after air exposure. At $530.3 \mathrm{eV}$ the $\mathrm{Bi}_{2} \mathrm{O}_{3}$ component increases in intensity after air exposure, ${ }^{39}$ further proof that part of the surface undergoes reoxidation. This increase is accompanied by a general decrease in intensity in the two components at $532.6 \mathrm{eV}$ (ref. 40 and 41) and $534.7 \mathrm{eV} ;{ }^{42}$ likely belonging to $\mathrm{O}-\mathrm{H} / \mathrm{S}-\mathrm{O}$ and $\mathrm{H}_{2} \mathrm{O}$ respectively. The hydroxyl arising from $\mathrm{H}$-termination of the oxidised areas of the sample, the sulfoxide from likely oxidation of the outer facing thiol groups and the water from adsorption on the layer of the reaction by-product due to hydrophilicity of the outer facing thiol group. ${ }^{\mathbf{4 3 4 4}}$ However, even after 10 days in air, there was no noticeable changes compared to a $1 \mathrm{~h}$ air exposure. Based on this data, an interdigitated "bilayer" of thiols is likely to be present; it
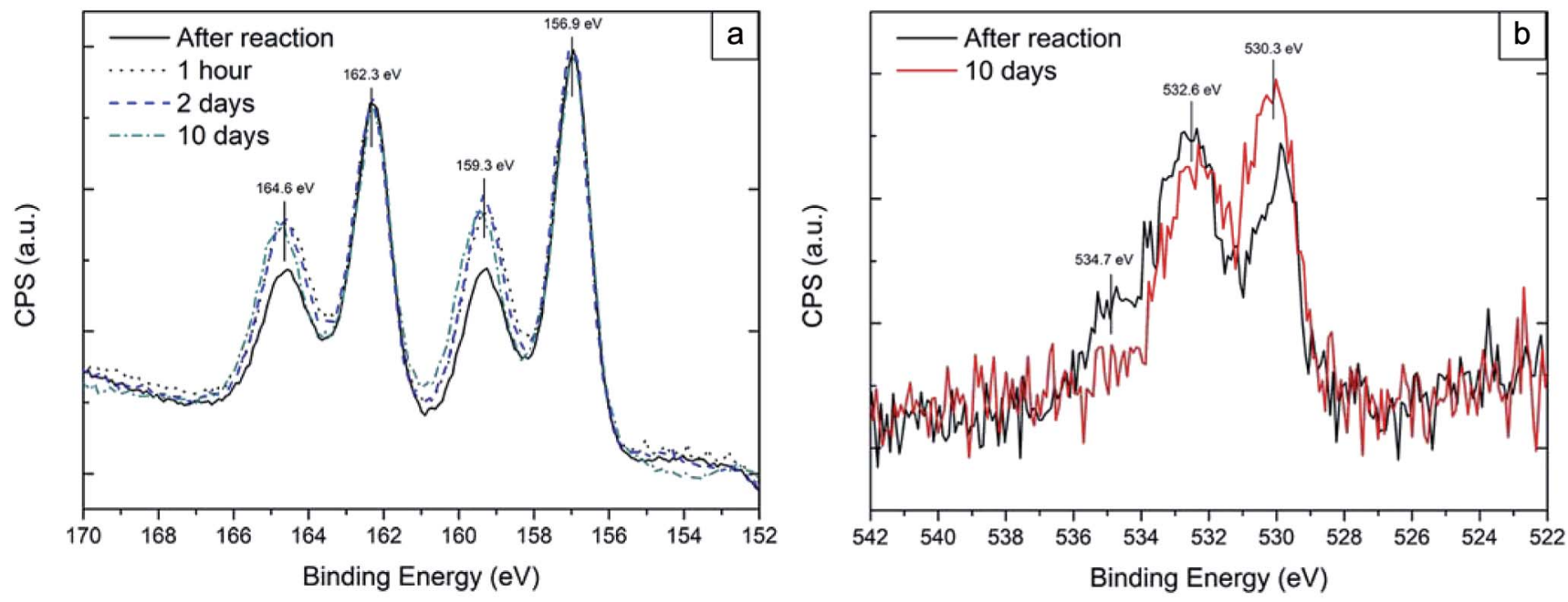

Fig. 4 (a) XPS spectra of Bi $4 \mathrm{f}$ core level at different times after functionalisation. Graphs have been normalised to the maximum of the sample after reaction to outline the reoxidation process. (b) $O 1$ s core level XPS spectra before and after reoxidation with main component peaks being highlighted. 
has already previously been shown that these "bilayer" structures can be easily formed both on nanoparticles ${ }^{45,46}$ and on 2-D films ${ }^{47}$ when using a polar solvent. Moreover, a previous study on silver colloidal nanoparticles ${ }^{48}$ has shown that an excess of precursor molecules can lead to bilayer structures which in turn lead to an increase in the ambient stability. In a similar fashion, $\mathrm{Cu}$ functionalisation with excess thiols, leads to multilayer formation of self-assembled copper thiolate layers. ${ }^{34}$ This hypothesis is further corroborated by the thickness measurements which has quantified a total carbon layer thickness of $4.3 \pm 0.5 \mathrm{~nm}$, which equates to about 2.7 1-dodecanethiol monolayers. This configuration prevents further surface oxidation, thus allowing the bismuth to be more stable in air. In contrast, an unpassivated bismuth metal surface obtained by reactive ion etching (RIE), completely reoxidizes in air after a 2 min exposure with oxidation levels comparable to the as received sample. Bi powder samples were also functionalized under $\mathrm{N}_{2}$ in a flask under similar conditions. The XPS analysis showed that the total content of oxide was reduced, although not to the same extent as for the thin film samples (see Fig. S3†); this is because of the higher oxide surface area of the powder, which in turn requires a higher number of thiol molecules to for oxide removal. In addition, as it was not possible to disperse the powder during the reaction, the thiol solution did not completely cover all the oxide surface available for the reaction.

\section{Conclusions}

In conclusion, the data reported in this paper shows for the first time that oxide removal and stabilisation of nanoscale bismuth films is possible outside of a vacuum environment using a wet chemistry approach using the self-assembly of 1-dodecanethiol as a passivating agent. After $1 \mathrm{~h}$ there was some re-oxidation of the surface however after this point it was shown that the surface remained stable for up to 10 days, as opposed to $2 \mathrm{~min}$ for unpassivated surfaces. The mechanism of oxide removal took place through a bismuth oxide-catalysed thiol oxidation reaction, resulting in the exposure of bare Bi surfaces that were readily passivated by thiol molecules. While there is a basic understanding of the possible mechanisms behind the passivation reaction and oxidation resistance, there is still potential for improvement of the quality of the layer for example by employing different length thiol molecules as longer thiols have been shown to be more effective in lowering thin film re-oxidation rates. ${ }^{21}$ The easiness of the method and low cost of the precursors make it also potentially attractive for future large-scale production processes where scaling up will be required.

\section{Conflicts of interest}

There are no conflicts to declare.

\section{References}

1 Y. Hasegawa, Y. Ishikawa, T. Saso, H. Shirai, H. Morita, T. Komine and H. Nakamura, Phys. Rev. B: Condens. Matter Mater. Phys., 2006, 382, 140-146.
2 C. R. Ast and H. Höchst, Phys. Rev. B: Condens. Matter Mater. Phys., 2003, 67, 1131021-1131024.

3 P. Hofmann, Prog. Surf. Sci., 2006, 81, 191-245.

4 X. Sun, Z. Zhang and M. S. Dresselhaus, Appl. Phys. Lett., 1999, 74, 4005-4007.

5 Y. Lin, X. Sun and M. Dresselhaus, Phys. Rev. B: Condens. Matter Mater. Phys., 2000, 62, 4610-4623.

6 R. Hartman, Phys. Rev., 1969, 181, 1070-1086.

7 G. E. Smith, G. A. Baraff and J. M. Rowell, Phys. Rev., 1964, 135, A1118-A1124.

8 F. Gity, L. Ansari, C. König, G. A. Verni, J. D. Holmes, B. Long, M. Lanius, P. Schüffelgen, G. Mussler, D. Grützmacher and J. C. Greer, Microelectron. Eng., 2018, 195, 21-25.

9 L. Ansari, G. Fagas, J. P. Colinge and J. C. Greer, Nano Lett., 2012, 12, 2222-2227.

10 F. Gity, L. Ansari, M. Lanius, P. Schüffelgen, G. Mussler, D. Grützmacher and J. C. Greer, Appl. Phys. Lett., 2017, 110, 093111.

11 M. G. Hapase, V. B. Tare and A. B. Biswas, Acta Metall., 1967, 15, 131-133.

12 G. D. Palkar, D. N. Sitharamarao and A. K. Dasgupta, Trans. Faraday Soc., 1963, 59, 2634-2638.

13 K. Komorita, M. Nisio and T. Nishinaga, Shinku, 1978, 21, 196-202.

14 E. Puckrin and A. J. Slavin, Phys. Rev. B: Condens. Matter Mater. Phys., 1990, 42, 1168-1176.

15 P. J. Kowalczyk, D. Belic, O. Mahapatra, S. A. Brown, E. S. Kadantsev, T. K. Woo, B. Ingham and W. Kozlowski, Appl. Phys. Lett., 2012, 100, 151904.

16 P. Ardalan, C. B. Musgrave and S. F. Bent, Langmuir, 2009, 25, 2013-2025.

17 Q. Cai, B. Xu, L. Ye, Z. Di, S. Huang, X. Du, J. Zhang, Q. Jin and J. Zhao, Appl. Surf. Sci., 2015, 353, 890-901.

18 G. Collins, P. Fleming, S. Barth, C. O'Dwyer, J. J. Boland, M. A. Morris and J. D. Holmes, Chem. Mater., 2010, 22, 6370-6377.

19 P. W. Loscutoff and S. F. Bent, Annu. Rev. Phys. Chem., 2006, 57, 467-495.

20 T. Bürgi, Nanoscale, 2015, 7, 15553-15567.

21 G. K. Jennings, J. C. Munro, T. H. Yong and P. E. Laibinis, Langmuir, 1998, 14, 6130-6139.

22 M. Adamovski, A. Zajac, P. Gründler and G. U. Flechsig, Electrochem. Commun., 2006, 8, 932-936.

23 T. Romann, V. Grozovski and E. Lust, Electrochem. Commun., 2007, 9, 2507-2513.

24 W. Kern, J. Electrochem. Soc., 1990, 137, 1887-1892.

25 P. J. Cumpson and P. C. Zalm, Surf. Interface Anal., 2000, 29, 403-406.

26 C. J. Powell and A. Jablonski, Electron Effective-AttenuationLength Database, MD, Gaithersburg, 2011.

27 R. Nyholm, A. Berndtsson and N. Martensson, J. Phys. C: Solid State Phys., 1980, 13, L1091-L1096.

28 V. S. Dharmadhikari, S. R. Sainkar, S. Badrinarayan and A. Goswami, J. Electron Spectrosc. Relat. Phenom., 1982, 25, 181-189.

29 A. Nersasian and P. R. Johnson, J. Appl. Polym. Sci., 1965, 9, 1653-1668. 
30 J. M. Bothwell, S. W. Krabbe and R. S. Mohan, Chem. Soc. Rev., 2011, 40, 4649-4707.

31 M. Postel and E. Duñach, Coord. Chem. Rev., 1996, 155, 127144.

32 V. C. Holmberg and B. A. Korgel, Chem. Mater., 2010, 22, 3698-3703.

33 C. W. Jones, Applications of Hydrogen Peroxide and Derivatives, Royal Society of Chemistry, Cambridge, 1999.

34 H. Keller, P. Simak, W. Schrepp and J. Dembowski, Thin Solid Films, 1994, 244, 799-805.

35 G. G. Briand and N. Burford, Adv. Inorg. Chem., 2000, 50, 285-357.

36 H. A. Phillips and N. Burford, Inorg. Chem., 2008, 47, 24282441.

37 J. Jayabharathi, G. A. Sundari, V. Thanikachalam, P. Jeeva and S. Panimozhi, RSC Adv., 2017, 7, 38923-38934.

38 M. Kirihara, Y. Asai, S. Ogawa, T. Noguchi, A. Hatano and Y. Hirai, Synthesis, 2007, 3286-3289, DOI: 10.1055/s-2007990800.

39 L. z. Zhao and J. b. Zhang, Solid State Commun., 1994, 90, 709-712.
40 B. J. Lindberg, K. Hamrin, G. Johansson, U. Gelius, A. Fahlman, C. Nordling and K. Siegbahn, Phys. Scr., 1970, 1, 286-298.

41 J. J. Pireaux, J. Riga, R. Caudano, J. J. Verbist, J. Delhalle, S. Delhalle, J. M. André and Y. Gobillon, Phys. Scr., 1977, 16, 329-338.

42 J. Russat, Surf. Interface Anal., 1988, 11, 414-420.

43 L. H. Dubois and R. G. Nuzzo, Annu. Rev. Phys. Chem., 1992, 43, 437-463.

44 H. Rieley, G. K. Kendall, F. W. Zemicael, T. L. Smith and S. Yang, Langmuir, 1998, 14, 5147-5153.

45 H. Fan, Z. Chen, C. J. Blinker, J. Clawson and T. Alam, J. Am. Chem. Soc., 2005, 127, 13746-13747.

46 B. Nikoobakht and M. A. El-Sayed, Langmuir, 2001, 17, 63686374.

47 R. N. Ward, D. C. Duffy, P. B. Davies and C. D. Bain, J. Phys. Chem., 1994, 98, 8536-8542.

48 V. Patil, K. S. Mayya, S. D. Pradhan and M. Sastry, J. Am. Chem. Soc., 1997, 119, 9281-9282. 